\title{
Preparation of Naphthalene Molecularly Imprinted Absorbent and its adsorption for Naphthalene
}

\author{
JIA Dan 1, a , Liu Huimin ${ }^{1, b}$ and GU Dongyan ${ }^{2, c}$ \\ ${ }^{1}$ Department of Resource and environment, Jilin Institute of Chemical Technology, Jilin 132022, Jilin, \\ China \\ 2 YongJi County environmental protection agency, Yongji 132200, Jilin, China \\ ajiadan9011@163.com, b1057362775@qq.com, c436085431@qq.com
}

Keywords: Preparation; Naphthalene Molecularly Imprinted Absorbent; adsorption.

Abstract. A novel naphthalene molecularly imprinted material was prepared using a template immobilized on Silica gel matrix by surface imprinting technique using naphthol as template, 3-isocyana to propyltriehthoxysilane as monomer, ethyl silicate as crosslinking agent. FTIR spectrum was adopted for the characterization of the structure of the molecularly imprinted absorbent. The separation and preconcentration characteristics of the imprinted absorbents for naphthalene were researched by batch procedures. The naphthalene molecularly imprinted adsorbent showed high adsorption capacity and significant selectivity for naphthalene.

\section{Introduction}

Molecularly imprinted polymers (MIPs) are an artificially synthesized macromolecular material, which has prearrangement of structure and specific molecular recognition ability [1]. In the last few years, MIPs were widely used for the selective enrichment and pretreatment of target compounds existing in complex matrix [2-4]. And molecular imprinting technology has been expanded to the field of environmental analysis and even reaction media [5].

MIPs are prepared by copolymerization of a cross-linking agent with the complex formed from a template and polymerizable monomers that have functional groups specifically interacting with the template through covalent or non-covalent bonds. After the template is removed from the resulting polymer matrix, binding sites having the size and shape complimentary to the template are generated [6]. However, the MIPs synthesized by traditional methods exhibit high affinity and selectivity but poor site accessibility to the target molecules. The kinetics of the sorption/desorption process is unfavorable and the mass transfer is slow [7]. Recently, molecularly imprinted sol-gel materials have been extensively studied [8-9]. These materials with binding sites situated at the surface show many advantages including high selectivity, more accessible sites, fast mass transfer and binding kinetics [10].

In this study, activated silica gel was used as the supporter and naphthol as the analog-model compound for the selective recognition of Naphthalene. Naphthalene is often contained in environmental water and is now attracting attention as an endocrine disturbance. The purpose of this work was to prepare a new and simple molecularly imprinted silica gel sorbent and to apply in SPE coupled with HPLC for the determination of Naphthalene in water samples. The proposed method presented high selectivity and adsorption capacity for Naphthalene.

\section{Experimental}

Reagents and Materials:Tetraethylorthosilicate (TEOS) was obtained from Aldrich Chem. Co. (Germany). 3-isocyana to propyltriehthoxysilane was supplied from Wuhan University Chemical Factory. Silica gel (80-120 mesh) was supplied from Qingdao Ocean Chemical Company. Naphthalene and naphthol was obtained from aladdin reagent company. 
Apparatus : FT-IR spectra were recorded using an Nicolet-6700 spectrometer (Thermo Elemental, USA). The chromatographic system consisted of a Model 230 HPLC pump and a UV detector. The UV detector was operated at $254 \mathrm{~nm}$.

\section{Preparation of Naphthalene -Imprinted Sorbent.}

The silica gel surfaces were activated by refluxing $8 \mathrm{~g}$ of silica gel (80-120 mesh) with 60 mLof 6 mol. $\mathrm{L}^{-1}$ hydrochloric acid under stirring for $8 \mathrm{~h}$, then the activated silica gel was filtered and washed with ultra pure water to neutral and dried under vacuum at $70{ }^{\circ} \mathrm{C}$ for $8 \mathrm{~h}$.

To prepare the naphthalene-imprinted sorbent, $0.4408 \mathrm{~g}$ of naphthol was dissolved in $5 \mathrm{~mL}$ of methanol under stirring, and $2 \mathrm{~mL}$ of 3-isocyana to propyltriehthoxysilane was added into the mixture. After the solution was stirred and refluxed for $30 \mathrm{~min}, 4 \mathrm{~mL}$ of TEOS was added. Stirring for $5 \mathrm{~min}$, $1.0 \mathrm{~g}$ of activated silica gel and $1 \mathrm{~mL}$ of $1.0 \mathrm{~mol} \bullet \mathrm{L}^{-1} \mathrm{HAc}$ were added. The mixture was stirred for 12 $\mathrm{h}$ at room temperature.

The product was filtrated and dried at $100^{\circ} \mathrm{C}$ for $12 \mathrm{~h}$. The sorbent was washed with $25 \mathrm{~mL}$ of methanol and $25 \mathrm{mLof} 1.0 \mathrm{~mol} \cdot \mathrm{L}^{-1} \mathrm{HCl}$ under stirring for $2 \mathrm{~h}$ to remove naphthol. After filtration, the product was washed with $50 \mathrm{~mL}$ of mixture of methanol and $6 \mathrm{~mol} \cdot \mathrm{L}^{-1} \mathrm{HCl}$ and ultra pure water, neutralized with $0.05 \mathrm{~mol} \cdot \mathrm{L}^{-1} \mathrm{NaOH}$ and washed by ultra pure water again. Finally, the sorbent was dried at $100{ }^{\circ} \mathrm{C}$ for $12 \mathrm{~h}$. The non-imprinted sorbent was also prepared using an identical procedure without adding naphthol.

Sorption studies : $10 \mathrm{~mL}$ of various concentrations of naphthalene were dissolved in methanol, then $50 \mathrm{mg}$ of naphthalene imprinted silica sorbent was added. The mixture was shaken for $2 \mathrm{~h}$ at room temperature. After the solution was centrifuged, the concentrations of the naphthalene in the supernatants were determined by HPLC.

\section{Results and discussion}

Characterization of the FTIR Spectra FT-IR spectra were obtained from blank silica and naphthalene-imprinted sorbent (Fig. 1). The features around $1101.3 \mathrm{~cm}^{-1}$ indicate $\mathrm{Si}-\mathrm{O}-\mathrm{Si}$ stretching vibrations. The $-\mathrm{OH}$ bond stretching band of the sorbent were observed at $3200-3600 \mathrm{~cm}^{-1}$. Compared with blank silica, a characteristic feature of the naphthalene-imprinted and non-imprinted sorbents is $\mathrm{N}-\mathrm{H}$ band around $1563.6 \mathrm{~cm}^{-1}$ and $\mathrm{C}-\mathrm{H}$ band around $2976.70 \mathrm{~cm}^{-1}$.

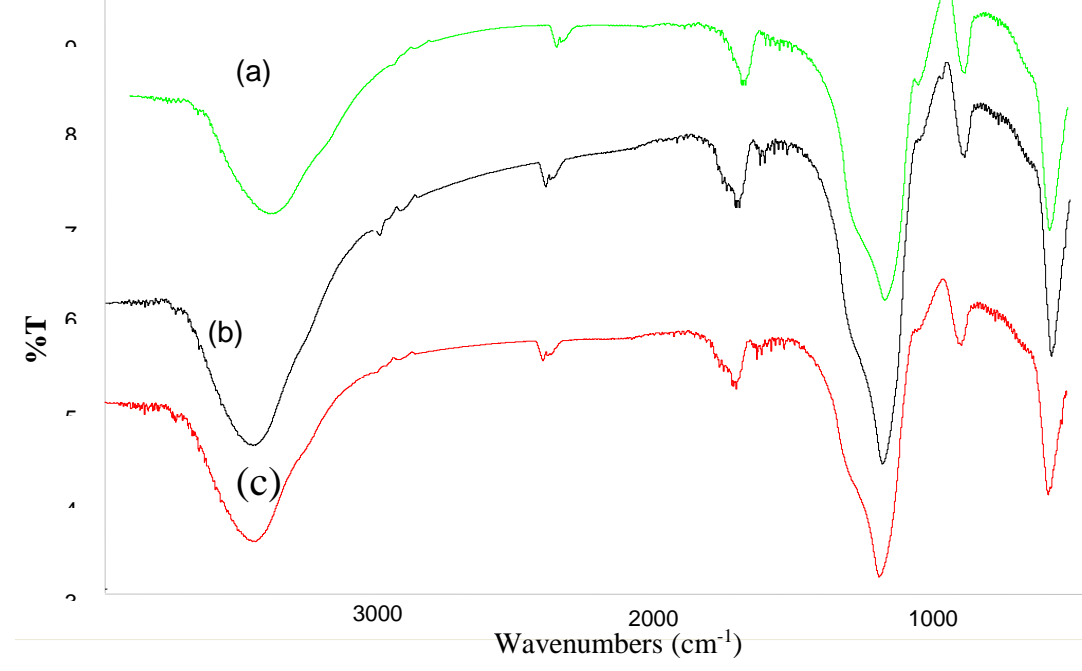

Fig. 1 FTIR spectra of (a) the blank silica; (b) the naphthalene -imprinted sorbent; and (c) non-imprinted sorbent.

\section{Adsorption capacity of the naphthalene-imprinted sorbent for naphthalene}

The adsorption capacity was an important factor to evaluate the MIPs. The range of naphthalene 
solution with the concentration of $100-1000 \mathrm{mg}_{\bullet} \mathrm{L}^{-1}$ was studied. As can be seen in Fig. 2, the amount of naphthalene adsorbed increased with the increase of the initial concentration of naphthalene solution. The static adsorption capacity of the naphthalene-imprinted silica gel sorbent and non-imprinted silica gel sorbent for naphthalene was calculated as 35.5 and $9.6 \mathrm{mg}_{\bullet} \mathrm{g}^{-1}$. The static adsorption capacity of the naphthalene-imprinted silica sorbent was much higher than that of the non-imprinted silica sorbent. So, the naphthalene-imprinted silica sorbent would be better to enrich trace naphthalene in the samples.

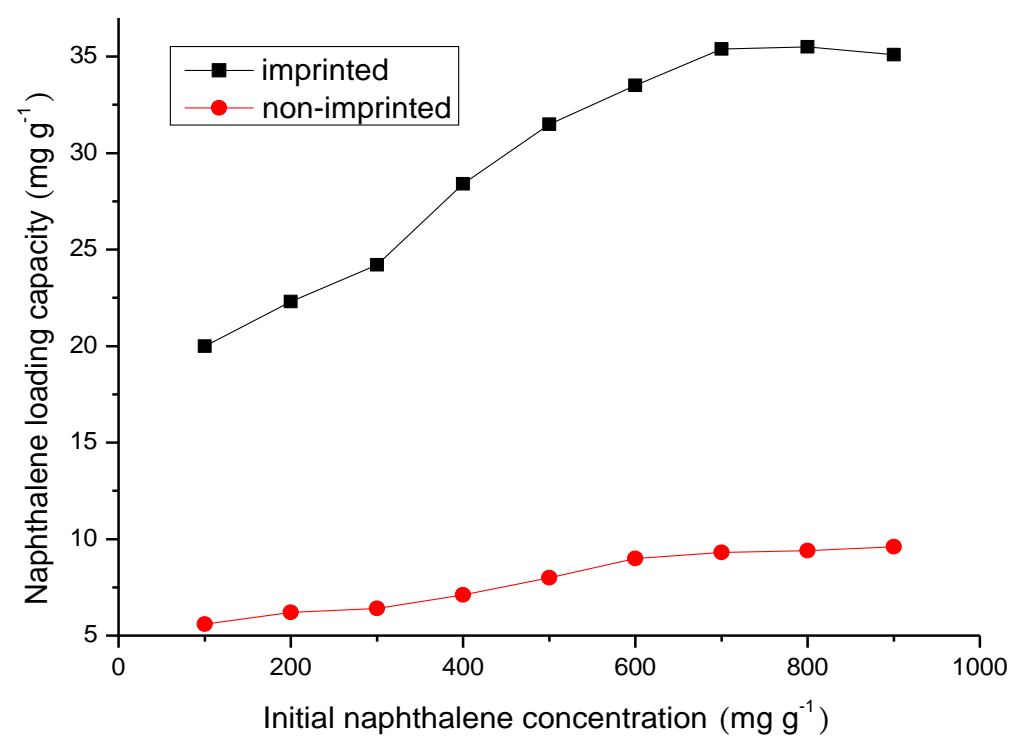

Fig. 2. Loading isotherm of naphthalene onto the imprinted and non-imprinted sorbents.

\section{Selectivity of the naphthalene-imprinted sorbent}

The structurally similar compound phenanthrene was chosen as the competitive species with naphthalene for the competitive recognition study. As can be seen in Table 1, distribution coefficient $(K d)$, selectivity coefficient of the sorbent $(k)$ and the relative selectivity coefficient $\left(k^{\prime}\right)$ was obtained in these competitive experiments. Distribution coefficient $(K d)$ suggested the character of a substance adsorbed by a sorbent, selectivity coefficient of the sorbent $(k)$ suggested the otherness of two substances adsorbed by one sorbent and relative selectivity coefficient $\left(k^{\prime}\right)$ suggested the otherness of two sorbents. These factors were calculated as the following formula (1)-(3).

$$
\begin{gathered}
K_{d}=\frac{C_{0}-C_{\mathrm{e}}}{C_{\mathrm{e}}} \times \frac{V}{M} \\
k=\frac{K_{d \text {-naphthalene }}}{K_{d \text {-phenanthrene }}} \\
k^{\prime}=\frac{k_{\text {imprinted }}}{k_{\text {nonimprinted }}}
\end{gathered}
$$

The $K_{\mathrm{d}}$ value of naphthalene on the imprinted and non-imprinted sorbents was both larger than that of phenanthrene. The $k^{\prime}$ value was 1.17 , which was higher than 1 and showed the naphthalene-imprinted silica sorbent had selectivity than the non-imprinted silica sorbent. 
Table 1 Competitive loading of naphthalene and phenanthrene by the naphthalene-imprinted and non-imprinted

\begin{tabular}{|c|c|c|c|c|}
\hline \multirow{2}{*}{ Sorbents } & \multicolumn{2}{|c|}{$K_{\mathrm{d}}\left(\mathrm{mL} \mathrm{g}^{-1}\right)$} & \multirow{2}{*}{$k$} & \multirow{2}{*}{$k^{\prime}$} \\
\hline & naphthalene & phenanthrene & & \\
\hline Imprinted & 66.67 & 17.69 & $\begin{array}{c}3.77 \\
-\end{array}$ & 1.17 \\
\hline Non-imprinted & 20.69 & 6.45 & 3.21 & \\
\hline
\end{tabular}

\section{Conclusions}

In this paper, a novel naphthalene-imprinted silica gel sorbent was synthesized by a surface molecular imprinting technique. The naphthalene-imprinted silica gel sorbent had high adsorption capacity, selectivity and good site accessibility for naphthalene. This new materials may be used as a solid-phase extraction sorbent to determine naphthalene in water samples or remove naphthalene from contaminated water.

\section{References}

[1] L.Q. Lin, J. Zhang, Q. Fu, L. C. He. Anal. Chim. Acta. Vol. 561 (2006), p. 178

[2] Y. Watabe, K. Hosoya, N. Tanaka, T. Kubo, T. Kondo, M. Morita. J. Chromatogr. A. Vol. 1073 (2005), p. 363.

[3] D. Lakshmi, B. B. Prasad, P.S. Sharma. Talanta Vol. 70 (2006), p.272

[4] F. Nie, J. Lu, Y. He, J. X. Du.Talanta Vol. 66 (2005), p. 728

[5] G. L. Yang, H.Y. Liu, M. M. Wang, S.B. Liu, Y. Chen. React. Function. Polym. Vol 66(2006), p.579

[6] R.J. Krupadam, M. S. Khan, S. R. Wate. Water Research. Vol. 44 (2010), p.681

[7] D. M. Han, G. Z. Fang, X.P.Yan. J. Chromatogr. A Vol. 1100 (2005), p.131

[8] A.Olwill, H. Hughes, M. O. Riordain, P. Mcloughlin. Biosens. Bioelectron. Vol. 20 (2004), p. 1045

[9] F. L. Dickert, O. Hayden. Anal. Chem. Vol. 74(2002), p. 1302

[10] A. Katz, M. E. Davis. Nature Vol.403 (2000), p. 286 